\section{Frailty, more than a word}

The word frailty comes from the Latin term fragilitas, which refers to something weak, something that can be easily broken, ruined or destroyed.

Frailty may vary depending on whether you are referring to an object or material, meaning little flexibility in its concrete form or inability to change its shape, or to the symbolic meaning, in this case, in relation to abstract concepts, such as emotional, psychological or social weakness.

At present, in the field of medicine, frailty may be defined as a complex biological disorder with decreased endurance and functional reserve, resulting in a greater vulnerability to adverse health conditions. Frailty is not necessarily a disability disorder, but they are not exclusive.

This concept has been widely studied in the elderly population as an apparent state of frailty under stress, mainly associated with aging, which affects homeostasis. ${ }^{1}$

In order to understand the starting points of this disorder, it is critical to identify the individuals at risk in advance so that intervention efforts are targeted at the most affected components.

This concept can be clearly implemented in adults and also in children with chronic conditions, as is the case of children with liver disease, whose severe alterations may involve their nutrition, endocrine system, immune status, and cognitive development.

The risk assessment of patients with endstage liver disease typically includes signs and symptoms, i.e., ascites, hepatosplenomegaly, deep jaundice, anemia, gastrointestinal bleeding, and biochemical alterations, such as reduced albumin levels, low serum sodium levels, decreased coagulation factors, etc.

In 1988, the United States National Institutes of Health included guidelines for cadaveric liver donation for children with severe liver disease to reduce the morbidity and mortality of transplant waiting list patients.

In February 2002, the United Network for Organ Sharing (UNOS) implemented the Pediatric End Stage Liver Disease (PELD) severity scoring system for organ allocation. The PELD score replaced urgency status and waiting list times.

The PELD score was rapidly implemented in different transplantation centers worldwide, including Argentina, where it has been used for several years now to establish organ allocation.

The PELD score was designed to reduce subjectivity and benefit waiting list patients who had a high probability of dying with a fast organ donation.

The system improved organ donation for high-risk patients; however, the PELD score was used in only $52 \%$ of cadaveric organ transplantation cases because a high percentage of such transplants in the US and Argentina were carried out through the request of a way of exception.

Since the PELD/MELD (Model for end-stage liver disease) scoring system minimizes waiting list times, many patients fail to reach a high score in time to receive an organ, leading to chronic impairment, especially in the pediatric population because of their nutritional status, neurocognitive development, and quality of life.

An interesting study about frailty in children with liver disease has been recently published; it assessed different factors that account for a reduced physiological function or homeostasis rupture. That study had a prospective, cross-sectional, multicenter design (17 liver transplantation centers) and assessed 71 children (5-17 years of age), 36 with compensated chronic liver disease and 35 with end-stage liver disease; all listed for liver transplantation. ${ }^{2}$

The study population was assessed based on the classic frailty phenotype proposed by Fried and using validated pediatric tools to determine each of the five classic alteration types: slowness, weakness, exhaustion, diminished physical activity, and shrinkage. After performing tests, scores were obtained; a score closer to 10 indicated a greater frailty status.

Results showed that subjects with end-stage liver disease had significantly higher frailty scores (median 5; IQR 4.7) than subjects with chronic liver disease (median 3; IQR 2.4); $(P<0.0001)$.

Those findings suggest that frailty scores capture a different factor of poor health in children assessed for transplant that is not considered by routine laboratory measures or nutritional tests for children with liver disease.

The elements that contributed to higher frailty scores in children with liver disease were exhaustion (fatigue), diminished physical activity, and a high prevalence of peripheral strength impairment. This last factor is very relevant in 
children with liver disease because it provides justification for future studies on psoas muscle area, whose reduction is known as sarcopenia, now considered a biological correlate of frailty and strength impairment. ${ }^{3}$

The problems of Lurz study were it was conducted in a small cohort and the tools used were targeted at children as of 5 years old, whereas most children requiring a transplant are younger. In the near future, probably, different questionnaires like the PedsQL may be used additionally to assess different conditions, such as fatigue, in younger children.

Such new open patient assessment is a good opportunity to establish a new chronic disease interpretation and, in addition, to understand their actual impairment status and plan strategies aimed at reducing risks.

Most likely, only very few people have been able to define the meaning of frailty as precisely as Jorge Luis Borges, who, in his "Poem of the Gifts," wrote: "Let no one reduce to tears or reproach, this statement of the mastery of God, who, with magnificent irony, gave me at once both books and night."
Daniel Eduardo D'Agostino, M.D. Pediatric Gastroenterology-Hepatology \& Intestinal and Liver Transplantation Division

Department of Pediatrics

Hospital Italiano de Buenos Aires

E-mail address: daniel.dagostino@hiba.org.ar

http: / / dx.doi.org/10.5546/aap.2019.eng.356

To cite: D'Agostino DE. Frailty, more than a word. Arch Argent Pediatr 2019;117(6):356-357.

\section{REFERENCES}

1. Fried LP, Tangen CM, Watson J, Newman AB, et al. Frailty in older adults: Evidence of a Phenotype. J Gerontol A Biol Sci Med Sci. 2001; 56(3):M146-56.

2. Lurz E, Quammie C, Englesbe M, Alonso E, et al. Frailty in Children with liver Disease: A Prospective Multicenter Study. J Pediatr. 2018; 194:109-15.e4.

3. Lurz E, Patel H, Frimpong RG, Ricciuto A, et al. Sarcopenia in Children With End-Stage Liver Disease. J Pediatr Gastroenterol Nutr. 2018; 66(2):222-6.

\section{The epistemology of introspection: the inner key to scientific research}

\section{The illusory subject-object separation}

Making a superficial analysis, it seems that a scientist and the object of their investigation are separate entities; however, an in-depth analysis clearly shows that this is not the case because an investigator and the object of investigation are part of a whole, where they play the everlasting game of interchangeability. For example, an investigator and the laboratory animal they are studying are joined by the exchange of air he/she is breathing, among other things; i.e., investigators are a self-aware fragment of the world capable of observing and analyzing another fragment of the world. ${ }^{1}$ In general, investigators believe that natural laws are discovered by observing and analyzing a world that is "external" to them. However, investigators actually re-discover laws that have always been a part of them because, being a fragment of the world, they are permeated by such laws., ${ }^{2,3}$ Therefore, the research process is a kind of instrument that contributes to elucidating natural laws that have always resided "inside" the investigator, thus facilitating the passage from the shadows of the unconscious to the light of the conscious. Investigators, as fractals of the universe, contain every secret about the universe, but such secrets are written in a natural (genetic-symbolic) language of shapes, sounds, and movements, common to the entire natural (inorganic and organic) world but that is illegible for human consciousness, whose language is that of words (signs). It is necessary to translate from one language to the other so that such elucidation takes place. These languages match, so they correspond to one another because words (conscious language) evolve out of symbols (unconscious language), which, in turn, evolve out of natural shapes, colors, and movements (natural language). Thus, for example, Newton's law of universal gravitation was described 